\title{
CCL22 and IL-37 inhibit the proliferation and epithelial-mesenchymal transition process of NSCLC A549 cells
}

\author{
YU-HUA CHEN $^{1 *}$, BI-YUN ZHOU ${ }^{2 *}$, XIAN-JING WU ${ }^{1}$, JUN-FA XU $^{3}$, JUN-AI ZHANG ${ }^{4}$, \\ YONG-HUA CHEN ${ }^{5}$ and SI-SI LIANG ${ }^{6}$
}

\begin{abstract}
Departments of ${ }^{1}$ Clinical Laboratory and ${ }^{2}$ Interventional Ward, Affiliated Hospital of Guangdong Medical College, Zhanjiang, Guangdong 524001; ${ }^{3}$ Department of Clinical Immunology, Institute of Laboratory Medicine, Guangdong Medical College, Dongguan 523808; ${ }^{4}$ Guangdong Provincial Key Laboratory of Medical Molecular Diagnostics, Dongguan 523808; Departments of ${ }^{5}$ Pathology and ${ }^{6}$ Orthopedics, Affiliated Hospital of Guangdong Medical College, Zhanjiang, Guangdong 524001, P.R. China
\end{abstract}

Received March 22, 2016; Accepted June 15, 2016

DOI: $10.3892 /$ or.2016.4995

\begin{abstract}
In the present study, we aimed to investigate the effects of CC chemokine ligand 22 (CCL22) and interleukin-37 (IL-37) on the proliferation and epithelial-mesenchymal transition (EMT) of non-small cell lung cancer (NSCLC) A549 cells. pDsRed-CCL22 and pEGFP-IL-37 plasmids were constructed. A549 cells were divided into six groups: the control, the pDsRed-N1 blank plasmid, the pEGFP-C1 blank plasmid, the pDsRed-CCL22 plasmid, the pEGFP-IL-37 plasmid and the pDsRed-CCL22 + pEGFP-IL-37 plasmid group. Expression levels and localization of CCL22 and IL-37 in cells were detected by confocal microscopy. Phase-contrast microscopy was applied for observing cellular morphology. Real-time fluorescence quantitative polymerase chain reaction (RT-qPCR) was used for detecting the mRNA levels of vimentin, $N$-cadherin and E-cadherin, and their protein expression levels were tested using western blotting. Constructed plasmids expressed CCL22 and IL-37, both of which had a co-localization in the cell membrane. MTT assay and cell observation results revealed that CCL22 and IL-37 inhibited the proliferation and EMT process of the A549 cells. The results of RT-qPCR and western blotting revealed that decreased vimentin and $\mathrm{N}$-cadherin mRNA and protein expression levels, and increased E-cadherin mRNA and protein expression levels were found
\end{abstract}

Correspondence to: Dr Yu-Hua Chen, Department of Clinical Laboratory, Affiliated Hospital of Guangdong Medical College, Zhanjiang, Guangdong 524001, P.R. China

E-mail: gdmccyh@163.com

*Contributed equally

Key words: CC chemokine ligand 22, interleukin-37, non-small cell lung cancer, epithelial-mesenchymal transition, A549, vimentin, $\mathrm{N}$-cadherin, E-cadherin in the pDsRed-CCL22 plasmid, pEGFP-IL-37 plasmid and pDsRed-CCL22 + pEGFP-IL-37 plasmid groups when compared with the control, the pDsRed-N1 blank plasmid and the pEGFP-C1 blank plasmid groups (all $\mathrm{P}<0.05$ ), and decreased vimentin and $\mathrm{N}$-cadherin mRNA and protein expression levels and increased E-cadherin mRNA and protein expression levels were found in the pDsRed-CCL22 + pEGFP-IL-37 plasmid group when compared with the pDsRed-CCL22 plasmid and the pEGFP-IL-37 plasmid groups (all $\mathrm{P}<0.05$ ). CCL22 and IL-37 with a co-localization in the A549 cells inhibited the proliferation and EMT process in A549 cells. The antitumor effects of CCL22 and IL37 provide a strategy for the treatment of NSCLC.

\section{Introduction}

Lung cancer continues to be the leading cause of cancer-related deaths worldwide due to its high incidence, malignant behavior and lack of major advancements in treatment strategy, accounting for $\sim 26 / 100,000$ patients in China $(1,2)$. Traditionally, lung cancer can be classified into two broad categories, including small cell lung cancer and non-small cell lung cancer (NSCLC) (3). Statistics show that NSCLC accounts for $85-90 \%$ of all lung cancers and is mainly comprised of squamous cell carcinoma, adenocarcinoma and large-cell carcinoma $(4,5)$. As a multifactorial cancer, NSCLC can be triggered by many risk factors including tobacco smoking, second-hand or passive smoking, diet, excess alcohol consumption, air pollution, occupational exposure and genetic predisposition $(6,7)$. A combination of radiotherapy and chemotherapy, along with surgical treatment, is the current treatment standard to achieve local tumor control and prolonged survival $(8,9)$. Notably, previous evidence has shown that the long-term survival rates of lung cancer patients remain low and the 5-year overall survival rate of NSCLC is $\sim 15 \%$ in the US and $<10 \%$ in China $(1,10,11)$. Therefore, there is a crucial need for finding prognostic markers to improve the clinical management of NSCLC patients. 

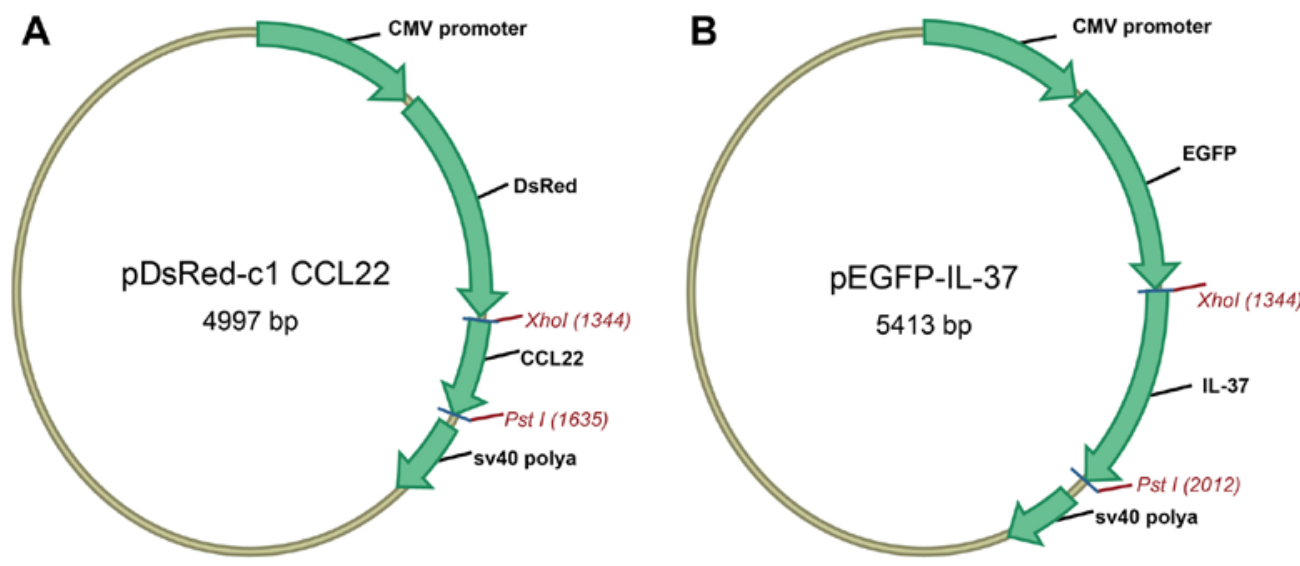

Figure 1. Plasmid profiles of (A) pDsRed-CCL22 and (B) pEGFP-IL-37.

To the best of our knowledge, a dysregulated inflammatory response is related to an increased risk of chronic disease and cancers (12). Chemokines, small pro-inflammatory chemotactic cytokines, play an important role in many tumor-related processes including growth, metastasis and angiogenesis (13). Chemokine (C-C motif) ligand 22 (CCL22), a member of the $\mathrm{CC}$ chemokine group, also known as macrophage-derived chemokine (MDC), is expressed by monocytes and dendritic cells (14). CCL22 has been demonstrated to play a homeostatic role in leukocyte trafficking in addition to its well-documented effects in innate immune cell activation and Th2 immunopathology, acting through the combination of CC-chemokine receptor 4 (CCR4) (15). Interleukin-37 (IL-37), also known as IL-1F7, is a newly discovered member of the interleukin family with anti-inflammatory and immune inhibitory effects, and is mainly expressed in the brain, heart, kidney, dendritic and peripheral blood of mononuclear cells $(16,17)$. Previous evidence revealed that IL-37 inhibits inflammation and immune reaction, and is found to be related to many diseases, such as acute myocardial infarction, sepsis and rheumatoid arthritis $(18,19)$. Furthermore, CCL22 also has functional effects on the susceptibility to inflammation, immunological diseases and malignancies $(20,21)$. As far as we know, the occurrence and development of lung cancer is closely related to inflammation (22), and few studies have been performed concerning the role and function of CCL22 and IL-37 in NSCLC. Thus, we conducted the present study to investigate the effects of CCL22 and IL-37 on the proliferation and the epithelial-mesenchymal transition (EMT) of NSCLC A549 cells.

\section{Materials and methods}

Plasmid construction. Polymerase chain reaction amplification was used for obtaining cDNA sequences of CCL22 and IL-37. cDNA sequences were recycled and inserted into pDsRed-N1 and pEGFP-C1 plasmids, respectively, via enzyme digestion of $X h o \mathrm{I}$ and $P s t \mathrm{I}$, to obtain pDsRedCCL22 and pEGFP-IL-37 expression plasmids. PCR conditions were: pre-denaturing at $95^{\circ} \mathrm{C}$ for $5 \mathrm{~min}, 95^{\circ} \mathrm{C}$ for $30 \mathrm{sec}, 58^{\circ} \mathrm{C}$ for $30 \mathrm{sec}$, and $72^{\circ} \mathrm{C}$ for $30 \mathrm{sec}$ with 25 cycles, and finally elongation at $72^{\circ} \mathrm{C}$ for $5 \mathrm{~min}$. The PCR primers were: CCL22 sense, $5^{\prime}$-cggctcgagatggatcgectacagactgcactc and antisense, 5'-cggctgcagtcattggctcagcttattg; IL-37 sense, 5'-cggctcgagatgtcctttgtgggggag and antisense, 5'-cggctgcaggcgttcaatggggcagtttc. Enzyme digestion was conducted at $37^{\circ} \mathrm{C}$ for 6 h. Fig. 1 shows the plasmid profiles.

Cell culture and grouping. The A549 cells (obtained from Shanghai Institute of Cell Biology of the Chinese Academy of Sciences, Shanghai, China, and retained in our laboratory) were cultured in $90 \%$ Dulbecco's modified Eagle's medium $($ DMEM) $+10 \%$ fetal bovine serum (FBS) (both from Thermo Fisher Scientific, Inc., Waltham, MA, USA). The A549 cells were divided into six groups in the present study: the control, the pDsRed-N1 blank plasmid, the pEGFP-C1 blank plasmid, the pDsRed-CCL22 plasmid, the pEGFP-IL-37 plasmid and the pDsRed-CCL22 + pEGFP-IL-37 plasmid groups. After being cultured for $24 \mathrm{~h}$, the cells were transfected with plasmids (total plasmid was $6 \mu \mathrm{g}$ ) via a Lipofectamine 3000 kit (Thermo Fisher Scientific).

Confocal microscopy detection. A coverglass coated with $0.1 \%$ gelatin was placed into a 6 -well plate, and then the above six groups of the A549 cells were added into the 6-well plate for culturing, respectively. When the cell density reached 80-90\% confluency, the medium was emptied and then the cells were washed three times with phosphate-buffered saline (PBS). Next, the cells were fixed with $4 \%$ paraformaldehyde for 15 min. Subsequently, 4',6-diamidino-2-phenylindole (DAPI) was also added to mark the nucleus. After being washed three times with PBS, the coverglass was inverted on a glass slide for observation under confocal microscopy (FV1000; Japan Olympus Corporation, Tokyo, Japan). pDsRed-CCL22 and pEGFP-IL-37 were observed after excitation at 558 and 490-nm wavelengths.

Methyl thiazolyl-tetrazolium (MTT) assay. A549 cells in six groups were cultured into a 96-well plate with a density of 5,000 cells in each well (each group has four duplicated wells). After being cultured for $24 \mathrm{~h}$, cell proliferation was tested at four time points $(24,48,72$ and $96 \mathrm{~h})$ via Vybrant ${ }^{\circledR}$ MTT Cell Proliferation Assay kit (Thermo Fisher Scientific).

Real-time fluorescence quantitative polymerase chain reaction (RT-qPCR). After being cultured for $72 \mathrm{~h}$, the cells were washed three times with PBS, and then general RNA extraction 
Table I. Primer sequences of vimentin, E-cadherin, N-cadherin and $\beta$-actin by RT-qPCR.

Sense

AATTTCACGCAGAGCAACAG

Vimentin

E-cadherin

$\mathrm{N}$-cadherin

$\beta$-actin
TTAACCTCACCAATCCTTGCT

AACCTAGCCTACTGGCCAAA

GCAGGGGGGAGCCAAAAGGGT
Antisense

CCACTAAGGCAGCACGTAAA

TAGCCCATTTCTTCCCAATC

AACATCGAGGTCGTAAACCC

TGGGTGGCAGTGATGGCATGG

RT-qPCR, real-time fluorescence quantitative polymerase chain reaction.

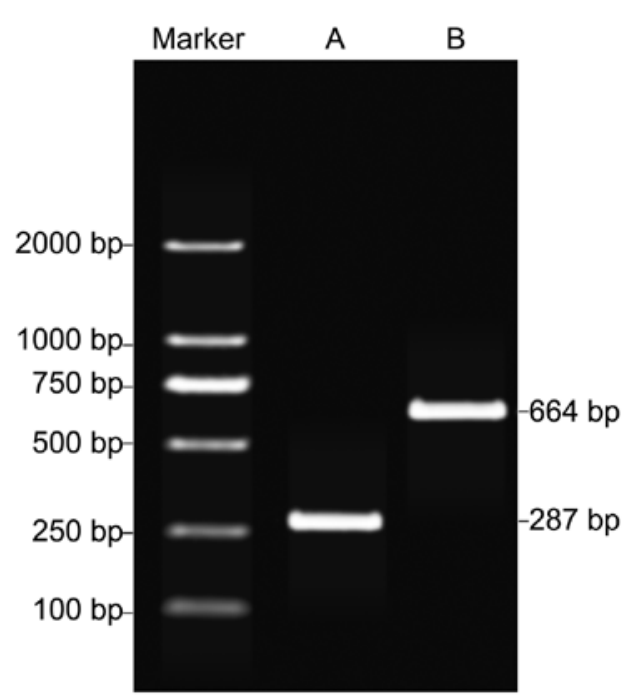

Figure 2. Enzyme digestion results of the pDsRed-CCL22 and pEGFP-IL-37 carrier (lane A, pDsRed-CCL22; lane B, pEGFP-IL-37).

via the RNAiso Plus kit (Takara, Bao Biological Engineering Co., Ltd., Dalian, China) followed. PrimeScript RT reagent kit (Takara, Bao Biological Engineering Co., Ltd.) and StepOnePlus (ABI) PCR system (Thermo Fisher Scientific) were applied for reverse transcription and RT-qPCR. The PCR system consisted of $1.6 \mu 1 \mathrm{cDNA}, 5 \mu \mathrm{l} 2 \mathrm{X}$ SYBR-Green Taq PCR Mix (Takara, Bao Biological Engineering Co., Ltd.), $0.2 \mu \mathrm{l}$ forward and $0.2 \mu \mathrm{l}$ reverse primers $(10 \mu \mathrm{M})$ and $3 \mu \mathrm{l}$ deuterium-depleted water (DDW). The reaction system was: pre-denaturing at $95^{\circ} \mathrm{C}$ for $5 \mathrm{~min}, 95^{\circ} \mathrm{C}$ for $10 \mathrm{sec}, 58^{\circ} \mathrm{C}$ for $10 \mathrm{sec}, 72^{\circ} \mathrm{C}$ for $10 \mathrm{sec}$, with a total of 60 cycles, and finally $72^{\circ} \mathrm{C}$ elongation for $10 \mathrm{~min}$. Vimentin (gene ID 7431), $N$-cadherin (gene ID 1000) and $E$-cadherin (gene ID 999) were detected in the present study, while $\beta$-actin (gene ID 2597) was regarded as the internal reference. Data analyses were conducted with the $2^{-\Delta \Delta \mathrm{Ct}}$ method, $2^{-\Delta \Delta \mathrm{Ct}}$ representing the multiple proportions between the test group and its control group. The formula was: $\Delta \Delta \mathrm{Ct}=\Delta \mathrm{Ct}_{\text {case group }}-\Delta \mathrm{Ct}_{\text {control group }}$, and $\Delta \mathrm{Ct}=\mathrm{Ct}_{\text {miRNA }}-\mathrm{Ct}_{\beta \text {-actin }}$. The primer sequences are listed in Table I.

Protein sample collection and western blotting. After $72 \mathrm{~h}$ of culture, the cells were washed, followed by the addition of RIPA lysis buffer (Thermo Fisher Scientific) and protease inhibitor (Sigma-Aldrich, St. Louis, MO, USA). After homogenization, the cells were centrifuged at $12,000 \times \mathrm{g}$ at $4{ }^{\circ} \mathrm{C}$ for $10 \mathrm{~min}$. The supernatant was gathered and used as the

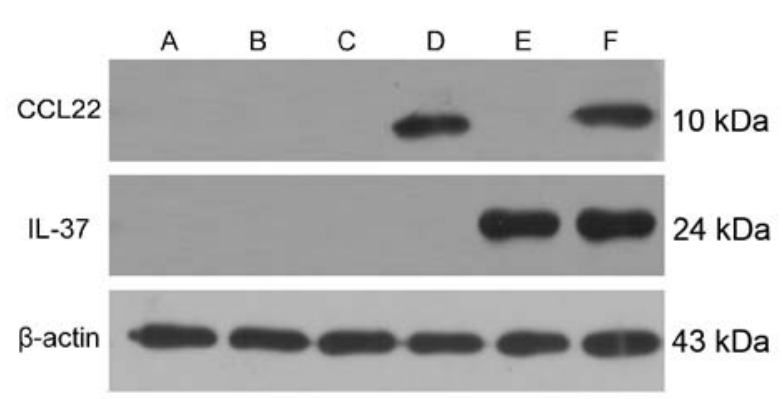

Figure 3. Expression levels of pDsRed-CCL22 and pEGFP-IL-37 fusion proteins in the A549 cells (lane A, the control; lane B, the pDsRedN1 blank plasmid; lane C, the pEGFP-C1 blank plasmid; lane D, the pDsRed-CCL22 plasmid; lane E, the pEGFP-IL-37 plasmid; lane F, the pDsRed-CCL22 + pEGFP-IL-37 group).

protein sample, and the concentration of protein was tested by bicinchoninic acid (BCA) assay. Then, the protein sample was stored at $-80^{\circ} \mathrm{C}$. Sodium dodecyl sulfate-polyacrylamide gel electrophoresis (SDS-PAGE) (10\%) was used for western blotting. To each well $20 \mu \mathrm{g}$ of protein sample was added and incubated overnight at $4^{\circ} \mathrm{C}$ with the use of rabbit polyclonal anti-vimentin, rabbit polyclonal anti-N-cadherin, rabbit polyclonal anti-E-cadherin (Abcam, Cambridge, UK) as primary antibodies. Horseradish peroxidase (HRP)-conjugated-goat anti-rabbit IgG (1:25,000) (Agrisera, Vännäs, Sweden) was regarded as the secondary antibody added into the samples. Then, the samples were incubated in a greenhouse for $30 \mathrm{~min}$, followed by the addition of HRP (Bio-Rad Laboratories, Inc., Hercules, CA, USA) for coloration. ImageQuant 350 and ImageQuant TL-1 (GE Healthcare, Logan, UT, USA) were applied for result analysis. $\beta$-actin was used as an internal reference.

Statistical analyses. Data analyses were performed using SPSS version 20.0 software (SPSS, Inc., Chicago, IL, USA). Continuous data are presented as the mean \pm SD. Comparisons between the two groups were conducted using a t-test, and comparisons among multiple groups by repeated measurements were performed using a one-way analysis of variance (ANOVA). $\mathrm{P}<0.05$ provided evidence of significant differences.

\section{Results}

Construction and detection of the pDsRed-CCL22 and pEGFP-IL-37 carrier. Fig. 2 shows the enzyme digestion 
A

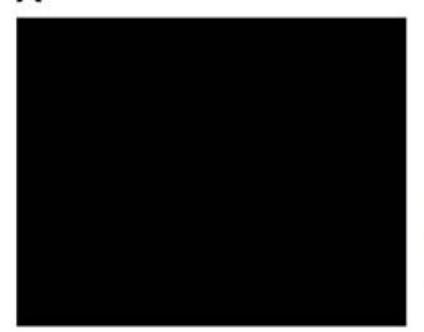

D

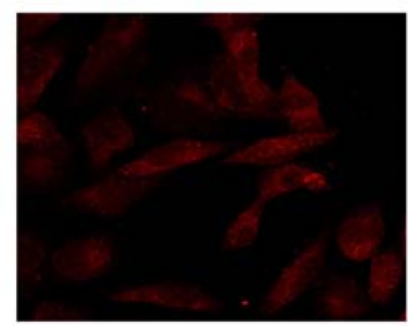

B

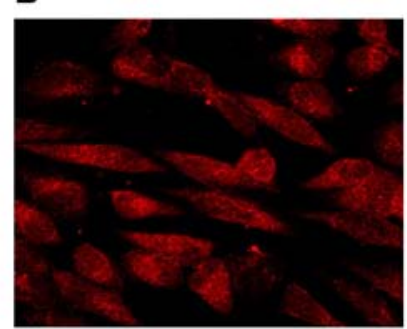

E

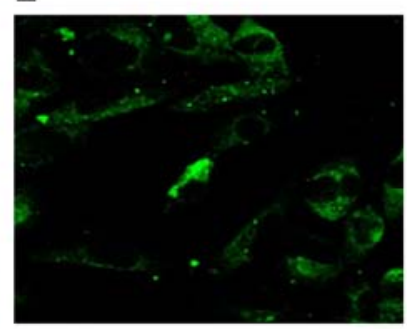

C

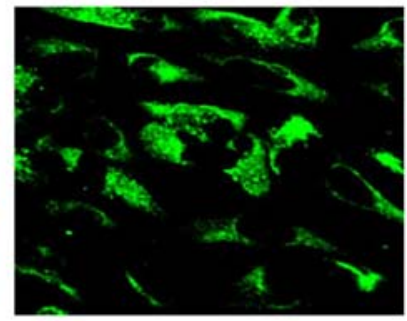

$\mathbf{F}$

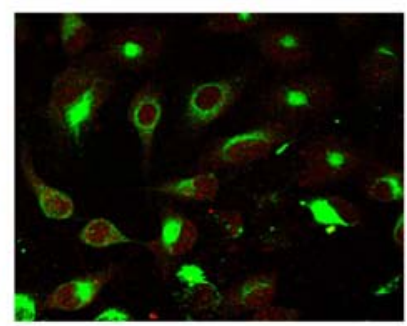

Figure 4. Localization of CCL22 and IL-37 in the cells was observed by confocal microscopy (A, the control; B, the pDsRed-N1 blank plasmid; C, the pEGFPC1 blank plasmid; D, the pDsRed-CCL22 plasmid; E, the pEGFP-IL-37 plasmid; and F, the pDsRed-CCL22 + pEGFP-IL-37 group).

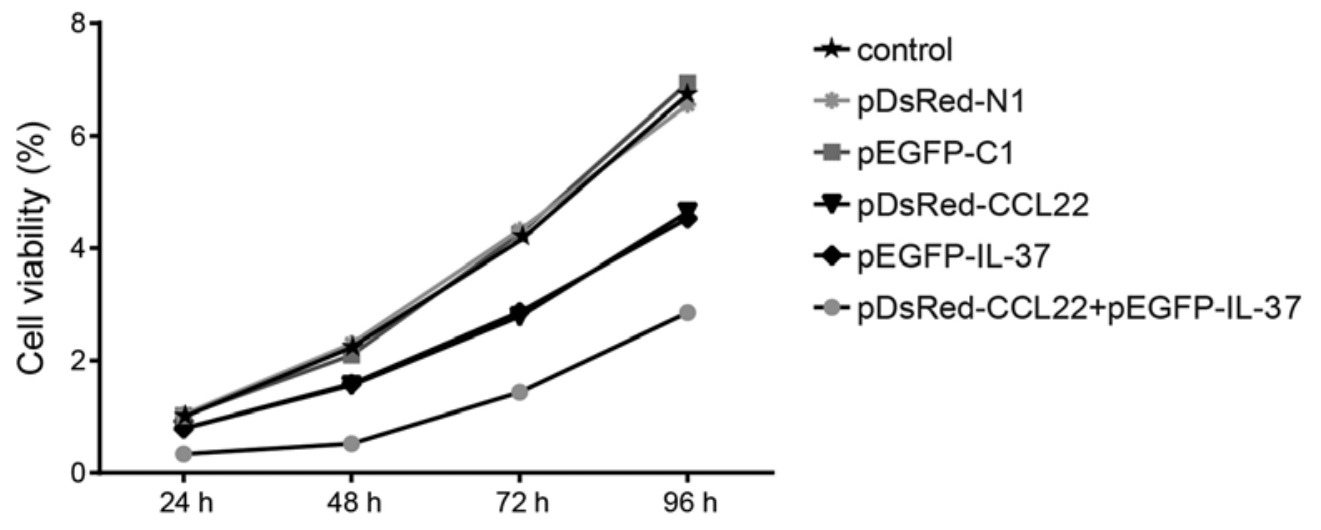

Figure 5. MTT assay was applied for assessmant of the proliferation of the A549 cells (MTT, methyl thiazolyl-tetrazolium).

results of the pDsRed-CCL22 and pEGFP-IL-37 carrier. The fragment lengths of pDsRed-CCL22 and pEGFP-IL-37 were 287 and $664 \mathrm{bp}$, respectively, after the enzyme digestion by XhoI and PstI, separately.

Expression levels of pDsRed-CCL22 and pEGFP-IL-37 fusion proteins in the A549 cells. Expression levels of pDsRed-CCL22 and pEGFP-IL-37 fusion proteins in the A549 cells were detected by western blotting. The results showed that no expression of CCL22 and IL-37 was found in the control, pDsRed-N1 blank plasmid and pEGFP-C1 blank plasmid groups; however, CCL22 was expressed in the pDsRed-CCL22 plasmid group, IL-37 was expressed in the pEGFP-IL-37 plasmid group, and CCL22 and IL-37 were both found simultaneously expressed in group 6 (Fig. 3).

Result of confocalmicroscopydetection. The results of confocal microscopy detection (Fig. 4) showed that no fluorescence was found in the control group, and fluorescence of the pDsRed-N1 blank plasmid and pEGFP-C1 blank plasmid groups was distributed in the cytoplasm. Expression of CCL22 and IL-37 proteins in the pDsRed-CCL22 plasmid and pEGFP-IL-37 plasmid groups, respectively, were mainly distributed in the cytoplasm. However, in the pDsRed-CCL22 + pEGFP-IL-37 plasmid group, CCL22 and IL-37 proteins were both found in the cytoplasm. These results revealed that CCL22 and IL-37 had a certain co-localization in the A549 cells.

Proliferation of the six cell groups via MTT assay. After being cultured for $24 \mathrm{~h}$, the activity of the A549 cells was detected every $24 \mathrm{~h}$. The relative activity of the cells in the control group was 1 at $24 \mathrm{~h}$. After 24, 48, 72 and $96 \mathrm{~h}$ of cell culture, no significant difference was found in the control, pDsRed-N1 blank plasmid and pEGFP-C1 blank plasmid groups (all $\mathrm{P}>0.05$ ). When compared with the control, pDsRed-N1 blank plasmid and pEGFP-C1 blank plasmid groups, lower cell proliferation was found in the pDsRed-CCL22 plasmid, pEGFP-IL-37 plasmid and pDsRed-CCL22 + pEGFP-IL-37 plasmid groups (all $\mathrm{P}<0.05$ ). However, the pDsRed-CCL22 + pEGFP-IL-37 plasmid group had lower cell proliferation when compared with the pDsRed-CCL22 plasmid and pEGFP-IL-37 plasmid groups alone (all $\mathrm{P}<0.05$ ) (Fig. 5). These results confirmed that CCL22 and IL-37 inhibited the proliferation of the A549 cells. 
A

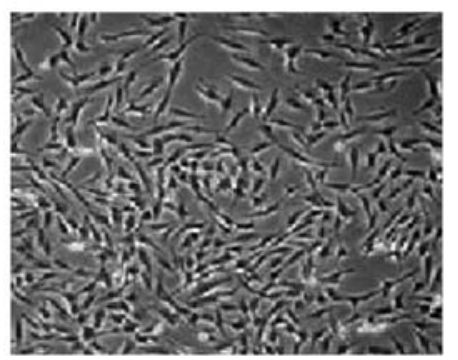

D

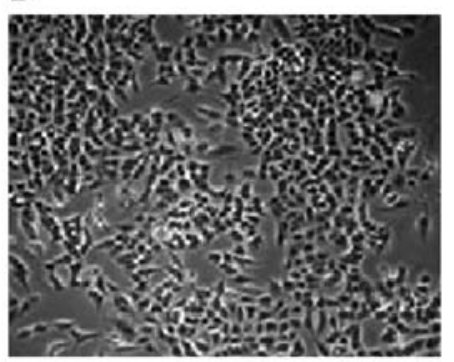

B

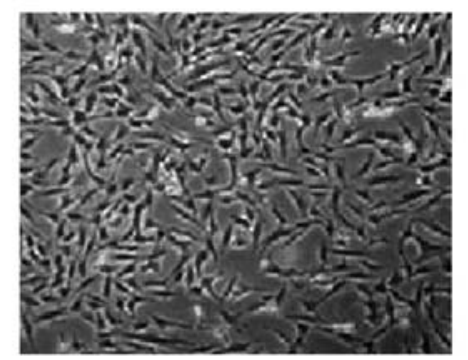

$\mathbf{E}$

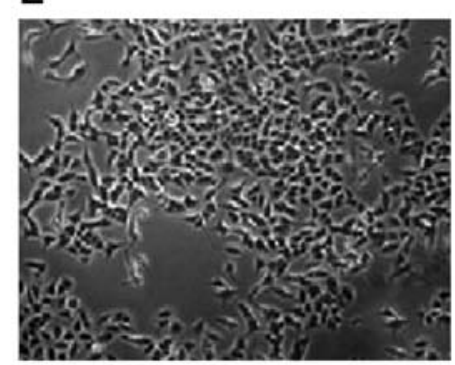

C

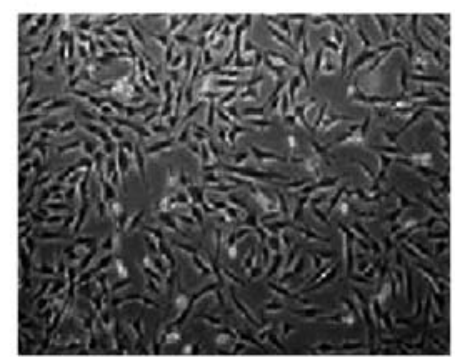

$\mathbf{F}$

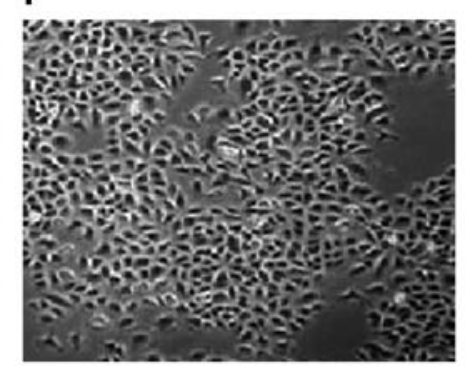

Figure 6. Morphological changes in the A549 cells during EMT were observed by microscopy (A, the control; B, the pDsRed-N1 blank plasmid; C, the pEGFPC1 blank plasmid; D, the pDsRed-CCL22 plasmid; E, the pEGFP-IL-37 plasmid; and F, the pDsRed-CCL22 + pEGFP-IL-37 plasmid group).

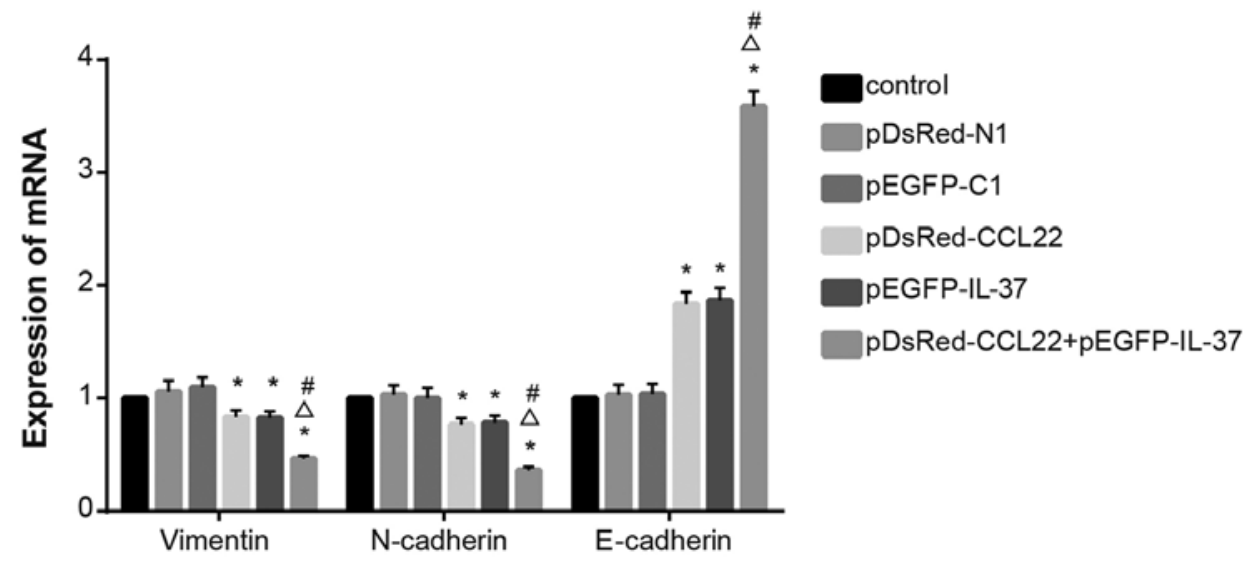

Figure 7. mRNA levels of EMT-related genes were detected by RT-qPCR (RT-qPCR, real-time fluorescence quantitative polymerase chain reaction). Control, the control group. " pDsRed-CCL22, pEGFP-IL-37 and pDsRed-CCL22 + pEGFP-IL-37 plasmid groups vs. the control group, P<0.05; ${ }^{\triangle}$ pDsRed-CCL22 + pEGFPIL-37 plasmid group vs. the pDsRed-CCL22 plasmid group, $\mathrm{P}<0.05$; ${ }^{*}$ pDsRed-CCL22 + pEGFP-IL-37 plasmid group vs. the pEGFP-IL-37 plasmid group, $\mathrm{P}<0.05$.

Cell morphologic observation under EMT. After $72 \mathrm{~h}$ of cell culture, the EMT process was observed under a microscope (Fig. 6). The results showed that obvious EMT was found in the control, pDsRed-N1 blank plasmid, and pEGFP-C1 blank plasmid groups. The cell morphologies were altered from pebble shaped cells into fusiform cells with elongated cell appearance. In addition, the cells were separated from peripheral cells, and the cells exhibited loose connections with larger intercellular space (Fig. 6A-C). EMT was also found in a few cells in the pDsRed-CCL22 plasmid and pEGFP-IL-37 plasmid groups. Cells showed fusiform shape and were loosely connected, but most of the cell morphology did not change significantly (still pebble shaped cells) (Fig. 6D and E). While, in the pDsRed-CCL22 plasmid + pEGFP-IL-37 plasmid group, epithelia maintained their pebble shape, tight junctions between cells and no EMT was noted (Fig. 6F). These results revealed that CCL22 and IL-37 inhibited the EMT process of the A549 cells and the inhibitory function was enhanced after the combination of CCL22 and IL-37.

mRNA levels of EMT-related genes. During EMT, the expression levels of vimentin and $N$-cadherin are increased, while the expression of E-cadherin is decreased. The results of RT-qPCR revealed that no significant difference was found when comparing vimentin, $N$-cadherin and $E$-cadherin among the control, the pDsRed-N1 blank plasmid, and the pEGFP-C1 blank plasmid groups $(\mathrm{P}>0.05)$. Decreased vimentin and $N$-cadherin mRNA expression levels, and increased $E$-cadherin mRNA expression were found in the pDsRed-CCL22 plasmid, the pEGFP-IL-37 plasmid and the pDsRed-CCL22 plasmid + pEGFP-IL-37 plasmid groups when compared with the control, the pDsRed-N1 blank plasmid and the pEGFP-C1 blank plasmid groups (all $\mathrm{P}<0.05$ ). Decreased vimentin and $N$-cadherin mRNA expression levels 

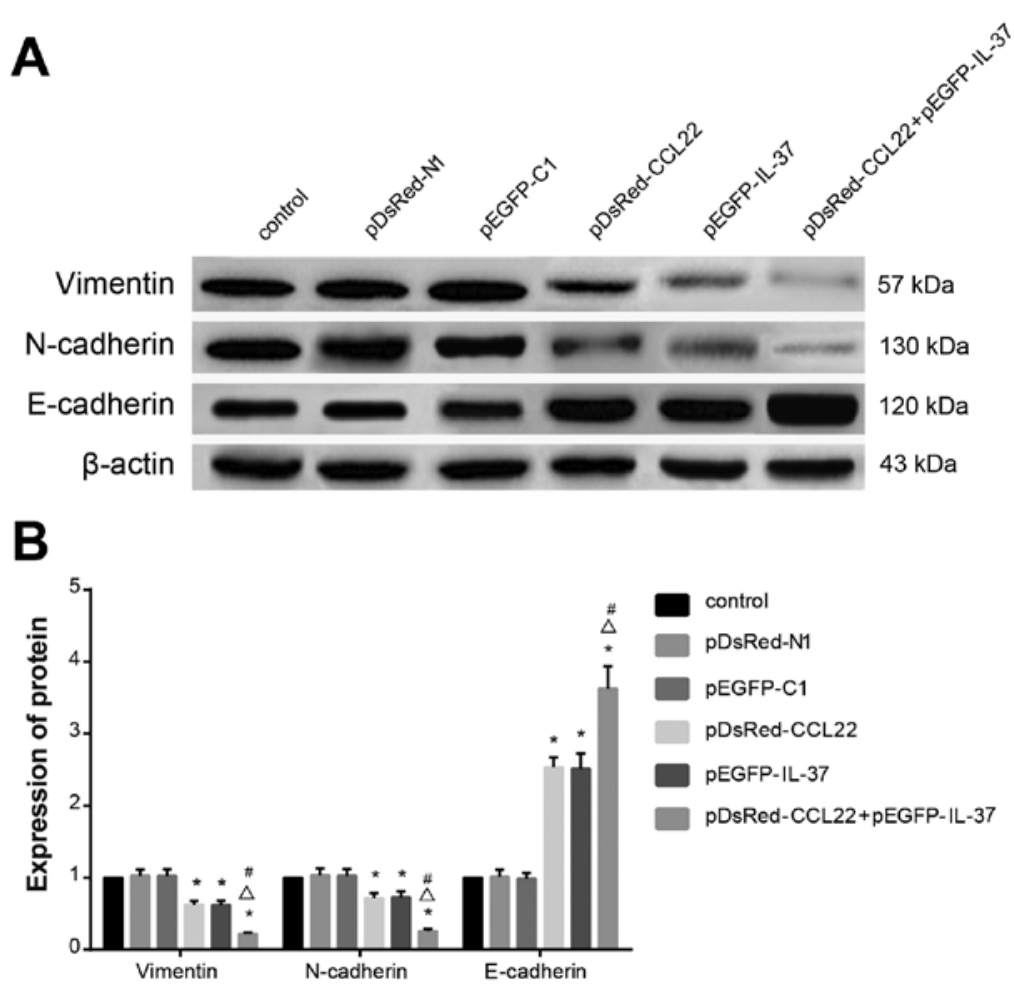

Figure 8. Protein expression levels of EMT-related genes were detected by western blotting. Control, the control. "pDsRed-CCL22 plasmid, pEGFP-IL-37 plasmid and pDsRed-CCL22 + pEGFP-IL-37 plasmid groups vs. the control group, $\mathrm{P}<0.05$; ${ }^{\wedge}$ pDsRed-CCL22 + pEGFP-IL-37 plasmid group vs. the pDsRedCCL22 plasmid group, $\mathrm{P}<0.05$; ${ }^{p}$ pDsRed-CCL22 + pEGFP-IL-37 plasmid group vs. the pEGFP-IL-37 plasmid group, $\mathrm{P}<0.05$.

and increased $E$-cadherin mRNA expression were found in the pDsRed-CCL22 plasmid + pEGFP-IL-37 plasmid groups when compared with the pDsRed-CCL22 plasmid and the pEGFP-IL-37 plasmid group (all P<0.05) (Fig. 7). These results showed that CCL22 and IL-37 inhibited the EMT process in the A549 cells.

Protein expression levels of EMT-related genes. The results from western blotting showed that no significant differences were found when comparing vimentin, $\mathrm{N}$-cadherin and E-cadherin protein expression levels among the control, pDsRed-N1 blank plasmid and pEGFP-C1 blank plasmid groups (all $\mathrm{P}>0.05$ ). Vimentin and $\mathrm{N}$-cadherin expression levels were decreased, and E-cadherin expression was increased in the pDsRed-CCL22 plasmid, pEGFP-IL-37 plasmid and pDsRed-CCL22 plasmid + pEGFP-IL-37 plasmid groups when compared with the control, pDsRed-N1 blank plasmid and pEGFP-C1 blank plasmid groups (all $\mathrm{P}<0.05$ ), and decreased vimentin and $\mathrm{N}$-cadherin expression levels and increased E-cadherin expression were found in the pDsRed-CCL22 plasmid + pEGFP-IL-37 plasmid groups when compared with the pDsRed-CCL22 plasmid and the pEGFP-IL-37 plasmid group (all $\mathrm{P}<0.05$ ) (Fig. 8). These results further confirmed that CCL22 and IL-37 inhibited the EMT process in the A549 cells.

\section{Discussion}

The present study represents a comprehensive analysis of CCL22 and IL-37 in regards to the proliferation and EMT of A549 cells. Our in-depth analyses concluded that CCL22 and IL-37 with co-localization in the A549 cells inhibited the prolif- eration and EMT process. The antitumor effects of CCL22 and IL37 provide a strategy for the treatment of NSCLC.

In the present study, we found that when compared with the control group, the pDsRed-N1 plasmid and the pEGFP-C1 plasmid group, lower cell proliferation was found in the pDsRed-CCL22 plasmid, the pEGFP-IL-37 plasmid and pDsRed-CCL22 plasmid + pEGFP-IL-37 plasmid groups; moreover, the pDsRed-CCL22 plasmid + pEGFP-IL-37 plasmid group had lower cell proliferation when compared with the pDsRed-CCL22 plasmid and the pEGFP-IL-37 plasmid group alone, suggesting that CCL22 and IL-37 can inhibit the proliferation of the A549 cells. Lung cancer is one of the typical inflammation-associated cancers, since smoking can lead to chronic inflammation (such as chronic obstructive pulmonary disease), and bronchioles and lungs are open organs which are more receptive to suffer the stimulation from a variety of physical factors that lead to inflammation (23). Chemokines represent a family of endogenous mediators of inflammation attracting various leukocyte subtypes to sites of infection and have been reported to affect malignancy in various ways, either by promoting or inhibiting tumor growth and metastasis formation via the activation of seven transmembrane domain receptors coupled to heterotrimeric $G$ proteins $(12,24)$. Previous evidence revealed that the binding of chemokines and their receptors can induce aggregation of actin, and regulate cell movement and migration (25). As far as we know, the main source of CCL22 is dendritic cells, which are the most powerful antigen-presenting cells in vivo, and capable of stimulating T cells into Th1 and Th2; and Th1 can secret interferon- $\gamma$ and inhibit CCL22, thereby inhibiting the proliferation of tumor cells (26). IL-37 is a cytokine which 
plays an important role in inflammation, autoimmunity, and other immunological disorders, and is synthesized as a protein which is processed to its mature form after stimulation (27). It has also been reported that IL-37 has the ability to kill microorganisms and increase production of several cytokines for the expansion of the acquired immune function (28). One study also demonstrated that IL-37 downregulated inflammation in a mouse model (29). Mature IL-37 can bind to Smad3 to regulate the key enzymes of multiple signaling pathways, including focal adhesion kinase (FAK), proline-rich tyrosine kinase (Pyk2), MAP kinase p38 $\alpha$, signal transducer and activator of transcription (STAT) p53 and mTOR. The expression of these key enzymes is downregulated and these pathways have been confirmed to be related to cell proliferation and migration (17).

Furthermore, we also found that vimentin and $\mathrm{N}$-cadherin expression levels were decreased, and E-cadherin expression was increased in the groups transfected with CCL22, IL-37 or both CCL22 and IL-37, suggesting that CCL22 and IL-37 can inhibit the EMT process in A549 cells. EMT was first described in the 1980's, and it was reported that EMT is not only a key biological process during embryonic morphogenesis, but also one of the earliest steps in solid tumor progression. EMT is related to tumor growth, metastasis as well as invasion and results in the conversion of tumors from low- to high-grade malignancy (30). E-cadherin, a crucial mediator of EMT, has been proven to result in rapid cell growth and metastasis in most cell types by loss of cell adhesion when E-cadherin loses its function (31). Li and Liu demonstrated that macrophages and activated fibroblasts can release a large number of growth factors, chemokines and matrix proteases, and then activate multiple signaling pathways such as the Smads, the ERK-MAPK and the PI3K-AKT signaling pathways to initiate EMT (32). Thus, we suspect that CCL22 and IL-37 inhibit the EMT process via multiple signaling pathways, including the Smads, the ERK-MAPK and the PI3K-AKT signaling pathways. However, the underlying mechanism of CCL22 and IL-37 inhibition in the EMT process warrants additional confirmation by further study.

Collectively, the present study demonstrated that CCL22 and IL-37 with co-localization in the A549 cells inhibited the proliferation and the EMT process in the A549 cells. The antitumor effects of CCL22 and IL37 provide a strategy for the treatment of NSCLC. The data from the present study can be explored by studying the effects of cytokines on the invasion and metastasis of NSCLC and by considering different cell lines for further substantiation of the aforementioned results.

\section{Acknowledgements}

The present study was supported by grants from the National Natural Science Foundation of China (No. 30972779, 81273237 and 81102850), the Science and Technology Project of Zhanjiang (2016B01036) and the Guangdong Medical College Research Fund (No. M2014026). We would like to acknowledge the reviewers for their helpful comments on this study.

\section{References}

1. Aberle DR, Adams AM, Berg CD, Black WC, Clapp JD Fagerstrom RM, Gareen IF, Gatsonis C, Marcus PM and Sicks JD; National Lung Screening Trial Research Team: Reduced lung-cancer mortality with low-dose computed tomographic screening. N Engl J Med 365: 395-409, 2011.
2. Wu H, Qiao N, Wang Y, Jiang M, Wang S, Wang C and Hu L: Association between the telomerase reverse transcriptase (TERT) rs2736098 polymorphism and cancer risk: Evidence from a casecontrol study of non-small-cell lung cancer and a meta-analysis. PLoS One 8: e76372, 2013.

3. Xu J, Yin Z, Gao W, Liu L, Yin Y, Liu P and Shu Y: Genetic variation in a microRNA-502 minding site in SET8 gene confers clinical outcome of non-small cell lung cancer in a Chinese population. PLoS One 8: e77024, 2013.

4. Liu XH, Liu ZL, Sun M, Liu J, Wang ZX and De W: The long non-coding RNA HOTAIR indicates a poor prognosis and promotes metastasis in non-small cell lung cancer. BMC Cancer 13: 464, 2013.

5. Pao W and Girard N: New driver mutations in non-small-cell lung cancer. Lancet Oncol 12: 175-180, 2011.

6. Dela Cruz CS, Tanoue LT and Matthay RA: Lung cancer: Epidemiology, etiology, and prevention. Clin Chest Med 32: 605-644, 2011.

7. Raz DJ, Gomez SL, Chang ET, Kim JY, Keegan TH, Pham J, Kukreja J, Hiatt RA and Jablons DM: Epidemiology of non-small cell lung cancer in Asian Americans: Incidence patterns among six subgroups by nativity. J Thorac Oncol 3: 1391-1397, 2008.

8. Albain KS, Swann RS, Rusch VW, Turrisi AT III, Shepherd FA, Smith C, Chen Y, Livingston RB, Feins RH, Gandara DR, et al: Radiotherapy plus chemotherapy with or without surgical resection for stage III non-small-cell lung cancer: A phase III randomised controlled trial. Lancet 374: 379-386, 2009.

9. Kitano H, Chung JY, Ylaya K, Conway C, Takikita M, Fukuoka J, Doki Y, Hanaoka J and Hewitt SM: Profiling of phospho-AKT, phospho-mTOR, phospho-MAPK and EGFR in non-small cell lung cancer. J Histochem Cytochem 62: 335-346, 2014.

10. Lee SH, Suh IB, Lee EJ, Hur GY, Lee SY, Lee SY, Shin C, Shim JJ, In KH, Kang KH, et al: Relationships of coagulation factor XIII activity with cell-type and stage of non-small cell lung cancer. Yonsei Med J 54: 1394-1399, 2013.

11. Song G, Qin T, Liu H, Xu GB, Pan YY, Xiong FX, Gu KS, Sun GP and Chen ZD: Quantitative breath analysis of volatile organic compounds of lung cancer patients. Lung Cancer 67: 227-231, 2010.

12. Ma H, Shu Y, Pan S, Chen J, Dai J, Jin G, Hu Z and Shen H: Polymorphisms of key chemokine genes and survival of non-small cell lung cancer in Chinese. Lung Cancer 74: 164-169, 2011.

13. Singh S, Sadanandam A and Singh RK: Chemokines in tumor angiogenesis and metastasis. Cancer Metastasis Rev 26: 453-467, 2007.

14. Niens M, Visser L, Nolte IM, van der Steege G, Diepstra A, Cordano P, Jarrett RF, Te Meerman GJ, Poppema S and van den Berg A: Serum chemokine levels in Hodgkin lymphoma patients: Highly increased levels of CCL17 and CCL22. Br J Haematol 140: 527-536, 2008.

15. Freier CP, Kuhn C, Rapp M, Endres S, Mayr D, Friese K, Anz D and Jeschke U: Expression of CCL22 and infiltration by regulatory $\mathrm{T}$ cells are increased in the decidua of human miscarriage placentas. Am J Reprod Immunol 74: 216-227, 2015.

16. Tete S, Tripodi D, Rosati M, Conti F, Maccauro G, Saggini A, Cianchetti E, Caraffa A, Antinolfi P, Toniato E, et al: IL-37 (IL-1F7) the newest anti-inflammatory cytokine which suppresses immune responses and inflammation. Int J Immunopathol Pharmacol 25: 31-38, 2012.

17. Nold MF, Nold-Petry CA, Zepp JA, Palmer BE, Bufler P and Dinarello CA: IL-37 is a fundamental inhibitor of innate immunity. Nat Immunol 11: 1014-1022, 2010.

18. Xu D, Wang A, Jiang F, Hu J and Zhang X: Effects of interleukin-37 on cardiac function after myocardial infarction in mice. Int J Clin Exp Pathol 8: 5247-5251, 2015.

19. Xia L, Shen H and Lu J: Elevated serum and synovial fluid levels of interleukin-37 in patients with rheumatoid arthritis: Attenuated the production of inflammatory cytokines. Cytokine 76: 553-557, 2015.

20. Wang G, Yu D, Tan W, Zhao D, Wu C and Lin D: Genetic polymorphism in chemokine CCL22 and susceptibility to Helicobacter pylori infection-related gastric carcinoma. Cancer 115: 2430-2437, 2009.

21. Erfani N, Nedaei Ahmadi AS, Ghayumi MA and Mojtahedi Z: Genetic polymorphisms of CCL22 and CCR4 in patients with lung cancer. Iran J Med Sci 39: 367-373, 2014.

22. Dougan M, Li D, Neuberg D, Mihm M, Googe P, Wong KK and Dranoff G: A dual role for the immune response in a mouse model of inflammation-associated lung cancer. J Clin Invest 121: 2436-2446, 2011 
23. Jin Y, Xiong X, Su Y, Hu J and Tao X: Serum vascular endothelial growth factor levels in patients with non-small cell lung cancer and its relations to the micrometastasis in peripheral blood. J Huazhong Univ Sci Technolog Med Sci 29: 462-465, 2009.

24. Thelen M and Stein JV: How chemokines invite leukocytes to dance. Nat Immunol 9: 953-959, 2008.

25. Speyer CL and Ward PA: Role of endothelial chemokines and their receptors during inflammation. J Invest Surg 24: 18-27, 2011.

26. Xia S, Wei J, Wang J, Sun H, Zheng W, Li Y, Sun Y, Zhao H, Zhang S, Wen T, et al: A requirement of dendritic cell-derived interleukin-27 for the tumor infiltration of regulatory T cells. J Leukoc Biol 95: 733-742, 2014.

27. Boraschi D, Lucchesi D, Hainzl S, Leitner M, Maier E, Mangelberger D, Oostingh GJ, Pfaller T, Pixner C, Posselt G, et al: IL-37: A new anti-inflammatory cytokine of the IL-1 family. Eur Cytokine Netw 22: 127-147, 2011.
28. Ma P, Gu B, Xiong W, Tan B, Geng W, Li J and Liu H: Glimepiride promotes osteogenic differentiation in rat osteoblasts via the PI3K/Akt/eNOS pathway in a high glucose microenvironment. PLoS One 9: e112243, 2014.

29. Maloy KJ and Powrie F: Intestinal homeostasis and its breakdown in inflammatory bowel disease. Nature 474: 298-306, 2011.

30. Lindsey S and Langhans SA: Crosstalk of oncogenic signaling pathways during epithelial-mesenchymal transition. Front Oncol 4: 358, 2014.

31. Nagathihalli NS and Merchant NB: Src-mediated regulation of E-cadherin and EMT in pancreatic cancer. Front Biosci 17: 2059-2069, 2012.

32. Li MX and Liu BC: Epithelial to mesenchymal transition in the progression of tubulointerstitial fibrosis. Chin Med J 120: 1925-1930, 2007. 